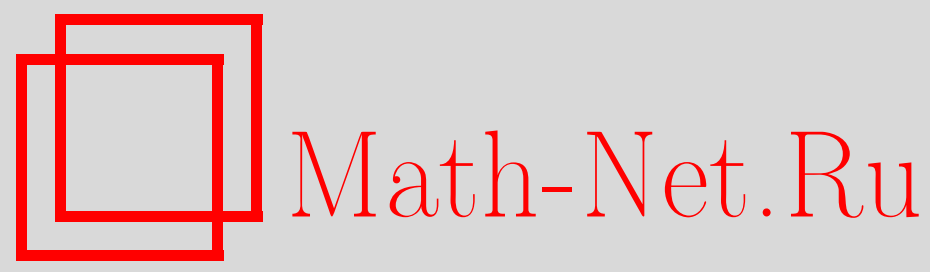

И. А. Рудаков, Периодические решения нелинейного волнового уравнения с непостоянными коэффициентами, $\mathrm{Ma}$ тем. заметки, 2004, том 76, выпуск 3, 427-438

DOI: https://doi.org/10.4213/mzm119

Использование Общероссийского математического портала Math-Net.Ru подразумевает, что вы прочитали и согласны с пользовательским соглашением http://www.mathnet.ru/rus/agreement

Параметры загрузки:

IP : 52.6 .47 .48

26 апреля 2023 г., $18: 17: 32$

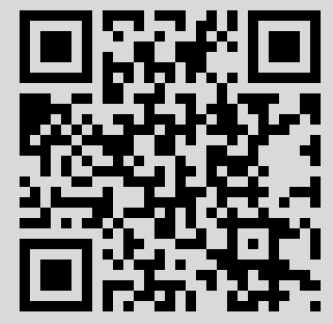


УДК 517.946

\section{ПЕРИОДИЧЕСКИЕ РЕШЕНИЯ \\ НЕЛИНЕЙНОГО ВОЛНОВОГО УРАВНЕНИЯ \\ С НЕПОСТОЯННЫМИ КОЭФФИЦИЕНТАМИ}

\section{И. А. Рудаков}

Доказано существование периодических по времени решений нелинейного уравнения вынужденных колебаний ограниченной струны. Оператор Даламбера имеет непостоянные коэффициенты. Нелинейное слагаемое имеет степенной рост.

Библиография: 12 названий.

1. Постановка задачи. В работе исследуется задача о периодических по времени решениях уравнения колебаний ограниченной неоднородной струны с нелинейным слагаемьг, имеющим степенной рост:

$$
\begin{gathered}
\rho(x) u_{t t}-\left(\rho(x) u_{x}\right)_{x}+|u|^{p-2} u=h(x, t), \quad 0<x<\pi, \quad t \in \mathbb{R}, \\
u(0, t)=u(\pi, t)=0, \quad t \in \mathbb{R}, \\
u(x, t+T)=u(x, t), \quad 0<x<\pi, \quad t \in \mathbb{R} .
\end{gathered}
$$

$\Phi$ ункция $\rho(x)$ удовлетворяет следующим условиям (см. [1]): $\rho(x) \in H^{2}(0, \pi)$,

$$
\beta_{0} \geqslant \rho(x) \geqslant \alpha_{0}>0 \quad \forall x \in(0, \pi)
$$

И

$$
\underset{[0, \pi]}{\operatorname{ess} \inf } \eta(x)>0
$$

где

$$
\eta(x)=\frac{1}{2} \frac{\rho^{\prime \prime}}{\rho}-\frac{1}{4}\left(\frac{\rho^{\prime}}{\rho}\right)^{2} .
$$

Константа $p$ в показателе степени удовлетворяет условию

$$
p>2 \text {. }
$$

Правая часть уравнения представляет собой заданную $T$-периодическую по времени функцию $h$, которая непрерывна на $[0, \pi] \times \mathbb{R}$.

Задача о периодических решениях нелинейного волнового уравнения с постоянньми коэффициентами $(\rho(x) \equiv 1)$ изучалась в классических работах [2]-[6] (см. также [7]). В 
работах [3], [6], [7] периодические решения получены с помощью теории степени отображения, как неподвижные точки соответствующих операторов. В статьях [2], [5] применяются аналоги леммы "горного перевала" Амбросетти-Рабиновича. В работе [4] используется метод расслоения С. И. Похожаева [8]. Статья [1] является одной из первых, в которой рассматривается волновое уравнение с непостоянными коэффищиентами. В ней доказано существование по крайней мере одного решения для случая, когда нелинейное слагаемое имеет "сублинейный” рост и удовлетворяет "глобальному" условию Липшица. В работе [9] удалось освободиться от условия Липшица.

В данной работе будет установлено существование периодических по времени решений волнового уравнения (1) с нелинейным слагаемым, имеющим степенной рост. Период времени $T$ соизмерим с $\pi$ и имеет вид $T=2 \pi / a$, где $a \in \mathbb{N}$.

Обозначим $\Omega=[0, \pi] \times[0, T], D=\left\{u(x, t) \in C^{\infty}(\Omega) \mid u(x, t)\right.$ удовлетворяет $(2),(3)$ и $\left.u_{t}(x, 0)=u_{t}(x, T)\right\}$. Определим

$$
\|u\|_{L_{r}(\Omega)}=\left\{\int_{\Omega} \rho(x)|u(x, t)|^{r} d x d t\right\}^{1 / r} \quad \forall u \in D
$$

где $r \geqslant 1$. Пространство $L_{r}(\Omega)$ является замыканием $D$ по норме $\|\cdot\|_{L_{r}(\Omega)}$. Пусть константа $q$ удовлетворяет условию $1 / p+1 / q=1$. Из (6) следует, что $1<q<2$. Для функций $u \in L_{p}(\Omega)$ и $v \in L_{q}(\Omega)$ определим

$$
(u, v)=\int_{\Omega} \rho(x) u(x, t) v(x, t) d x d t .
$$

ОПРЕДЕЛЕНИЕ. Функция $u \in L_{p}(\Omega)$ назьвается обобщенным решением задачи (1)-(3), если

$$
\int_{\Omega} u\left(\rho(x) \varphi_{t t}-\left(\rho(x) \varphi_{x}\right)_{x}\right) d x d t+\int|u|^{p-2} u \varphi d x d t=\int_{\Omega} h \varphi d x d t \quad \forall \varphi \in D .
$$

Целью настоящей работы является доказательство следующей теоремы.

ТЕОремА 1. Для любых положстельных чисел $d_{1}, d_{2}$ существует $a_{0}=a_{0}\left(d_{1}, d_{2}\right)$ $\in \mathbb{N}$ такое, что при любом $a \geqslant a_{0}$ и любой $T$-периодической по $t$ и непрерывной на $[0, \pi] \times \mathbb{R}$ функиии $h(x, t) c\|h\|_{C} \leqslant d_{1}$ задача (1)-(3) имеет обобщенное решение $u \in L_{p}(\Omega)$ mакое, что $\|u\|_{p} \geqslant d_{2}$.

Заменим, как в [5], задачу (1)-(3) на эквивалентную $2 \pi$-периодическую задачу. Для этого обозначим через $L_{r},\|\cdot\|$ соответственно $L_{r}(\Omega),\|\cdot\|_{L_{2}(\Omega)}$ при $a=1$. Введем новую неизвестную функцию

$$
v(x, t)=u\left(x, \frac{t}{a}\right) .
$$

Функция $u(x, t)$ является решением задачи $(1)-(3)$ тогда и только тогда, когда $v(x, t)$ является решением задачи

$$
\begin{gathered}
a^{2} \rho(x) v_{t t}-\left(\rho(x) v_{x}\right)_{x}+|v|^{p-2} v=f(x, t), \quad 0<x<\pi, \quad t \in \mathbb{R}, \\
v(0, t)=v(\pi, t)=0, \quad t \in \mathbb{R}, \\
v(x, t+2 \pi)=v(x, t), \quad 0<x<\pi, \quad t \in \mathbb{R} .
\end{gathered}
$$

Здесь $f(x, t)=h(x, t / a)$. 
2. Свойства линейной части уравнения. Обозначим $\lambda_{n}$ и $\varphi_{n}=\varphi_{n}(x)$ соответственно собственные значения и собственные функции задачи Штурма-Лиувилля

$$
-\left(\rho \varphi_{n}^{\prime}\right)_{x}=\rho \lambda_{n}^{2} \varphi_{n}, \quad \varphi_{n}(0)=\varphi_{n}(\pi)=0, \quad n \in \mathbb{N} .
$$

Здесь $\varphi^{\prime}=\varphi_{x}=d \varphi / d x$. Систему функций $\varphi_{n}(x)$ можно считать ортонормированной в $L_{2}(0, \pi)$ со скалярным произведением

$$
(\varphi, \psi)=\int_{[0, \pi]} \rho(x) \varphi(x) \psi(x) d x, \quad \varphi, \psi \in L_{2}(0, \pi) .
$$

В работе [1] доказано, что если выполнены условия (4), (5), то существуют константы $b_{0}, b_{1}>0$ такие, что

$$
\lambda_{n}=n+\theta_{n}
$$

где

$$
0<b_{0} \frac{1}{n} \leqslant \theta_{n} \leqslant b_{1} \frac{1}{n} \quad \forall n \in \mathbb{N} .
$$

Рассмотрим полную, ортонормированную в $L_{2}(\Omega)$ систему функций

$$
\left\{\frac{1}{\sqrt{2 \pi}} \varphi_{n}(x), \frac{1}{\sqrt{\pi}} \varphi_{n}(x) \cos (m t), \frac{1}{\sqrt{\pi}} \varphi_{n}(x) \sin (m t)\right\}_{m, n \in \mathbb{N}} .
$$

Определим оператор $A_{0}: L_{2}(\Omega) \rightarrow L_{2}(\Omega)$ так, что

$$
D\left(A_{0}\right)=\left\{u \in C^{2}(\bar{\Omega}) \mid u=\sum_{n=1}^{N} \sum_{m=0}^{M} \varphi_{n}(x) \cdot\left(a_{n m} \cos (m t)+b_{n m} \sin (m t)\right), M, N \in \mathbb{N}\right\}
$$

и $A_{0} \varphi=a^{2} \rho \varphi_{t t}-\left(\rho \varphi_{x}\right)_{x} \forall \varphi \in D\left(A_{0}\right)$. Обозначим

$$
\bar{A}_{0} \varphi=\frac{1}{\rho} A_{0} \varphi \quad \forall \varphi \in D\left(A_{0}\right) .
$$

Функции (12) являются собственными функциями оператора $\bar{A}_{0}$ с собственньми значениями

$$
\mu_{n m}=\lambda_{n}^{2}-(a m)^{2}=\left(n+\theta_{n}\right)^{2}-(a m)^{2},
$$

где $n \in \mathbb{N}, m \in \mathbb{Z}_{+}=\mathbb{N} \cup\{0\}$.

Пусть $A$ есть оператор в $L_{2}(\Omega)$, являющийся замыканием по графику оператора $\bar{A}_{0}$. Выделим среди множества собственных функций оператора $A$ два подмножества:

$$
\begin{aligned}
& \Lambda_{1}=\left\{\frac{1}{\sqrt{\pi}} \varphi_{n}(x) \cos \left(\frac{n}{a} t\right), \frac{1}{\sqrt{\pi}} \varphi_{n}(x) \sin \left(\frac{n}{a} t\right) \mid \frac{n}{a} \in \mathbb{N}\right\}, \\
& \Lambda_{2}=\left\{\varphi_{n}(x) \cos (m t), \varphi_{n}(x) \sin (m t) \mid(n, m) \in M\right\},
\end{aligned}
$$

где $M=\left\{(n, m) \in \mathbb{N} \times \mathbb{Z}_{+} \mid \lambda_{n} \neq a m, n \neq a m\right\}$.

Представим $L_{2}(\Omega)$ в виде прямой суммы подпространств

$$
L_{2}(\Omega)=N_{1} \oplus N_{2} \oplus N_{3},
$$


где $N_{1}=N(A)$ есть ядро $A, N_{2}=\overline{L\left(\Lambda_{1}\right)}$ (замыкание в $L_{2}(\Omega)$ линейной оболочки $\Lambda_{1}$ ), $N_{3}=\overline{L\left(\Lambda_{2}\right)}$.

Заметим, что на пространстве $N_{2}$ собственные значения оператора $A$ равны

$$
2 n \cdot \theta_{n}+\theta_{n}^{2}>2 b_{0}>0 .
$$

Перечислим свойства оператора $A$, установленные в работе [1]:

а) $A$ - самосопряженньй оператор, образ $R(A)$ замкнут в $L_{2}(\Omega)$ и

$$
A^{-1} \in L(R(A), R(A))
$$

б) ядро $N(A)$ конечномерно, а если

$$
\frac{2}{\pi} \int_{0}^{\pi} \eta(x) d x<3
$$

то $N(A)=\{0\}$

в) $R(A)=N(A)^{\perp}, L_{2}(\Omega)=N(A) \oplus R(A)$.

Докажем еще некоторые свойства оператора $A$.

Лемма 1. Существует $a_{1} \in \mathbb{N}$ такое, что для всех натуральных $a \geqslant a_{1}$ имеет место оценка

$$
\left\|A^{-1} v\right\|_{\infty} \leqslant C_{0}\|v\|_{\alpha} \quad \forall v \in N_{3}
$$

где $\alpha>1$ и $C_{0}=C_{0}\left(a_{1}\right)$ - константа, не зависящая от $v и а$.

ДокАЗАтЕльСтво. Рассмотрим вначале случай $\alpha \in(1,2]$. Пусть $\left\|\varphi_{n}(x)\right\|_{\infty} \leqslant C_{1}$ $\forall n$ и $1 / \alpha^{\prime}+1 / \alpha=1$. Пусть $\bar{v}_{n m}, \overline{\bar{v}}_{n m}$ есть коэффициенты Фурье функции $v$ по системе $\Lambda_{2}$. Используя неравенство Гёльдера и Хаусдорфа-Юнга, выведем

$$
\begin{aligned}
\left\|A^{-1} v\right\|_{\infty} & \leqslant C_{2} \sum_{(n, m) \in M}^{\infty} \frac{\left|\bar{v}_{n m}\right|+\left|\overline{\bar{v}}_{n m}\right|}{\left|\lambda_{n}^{2}-(a m)^{2}\right|} \\
& \leqslant C_{2}\left(\sum_{(n, m) \in M}\left(\left|\bar{v}_{n m}\right|^{\alpha^{\prime}}+\left|\overline{\bar{v}}_{n m}\right|^{\alpha^{\prime}}\right)\right)^{1 / \alpha^{\prime}} \cdot\left[\sum_{(n, m) \in M}\left|\lambda_{n}^{2}-(a m)^{2}\right|^{-\alpha}\right]^{1 / \alpha} \\
& \leqslant C_{3}\|v\|_{\alpha} \cdot\left[\sum_{(n, m) \in M}\left|\lambda_{n}^{2}-(a m)^{2}\right|^{-\alpha}\right]^{1 / \alpha}
\end{aligned}
$$

Рассмотрим числовой ряд

$$
\begin{aligned}
I & =\sum_{(n, m) \in M}\left|\lambda_{n}^{2}-(a m)^{2}\right|^{-\alpha} \\
& =\sum_{\substack{(n, m) \in M \\
m \neq 0}}\left|n^{2}-(a m)^{2}+2 n \theta_{n}+\theta_{n}^{2}\right|^{-\alpha}+\sum_{n=1}^{\infty} \frac{1}{\left(n+\theta_{n}\right)^{2 \alpha}} \\
& <\sum_{n=1}^{\infty} \frac{1}{n^{2 \alpha}}+\sum_{\substack{(n, m) \in M \\
m \neq 0}}\left|n^{2}-(a m)^{2}\right|^{-\alpha}\left|1+\frac{2 n \theta_{n}+\theta_{n}^{2}}{n^{2}-(a m)^{2}}\right|^{-\alpha} .
\end{aligned}
$$


Обозначим через $C_{4}=2 b_{1}+\max \theta_{n}^{2}$. Тогда

$$
\left|\frac{2 n \theta_{n}+\theta_{n}^{2}}{n^{2}-(a m)^{2}}\right| \leqslant \frac{C_{4}}{n+a m},
$$

так как $(n, m) \in M$. Следовательно, множество

конечное и существует

$$
S=\left\{(n, m) \in M|| \frac{2 n \theta_{n}+\theta_{n}^{2}}{n^{2}-(a m)^{2}} \mid>\frac{1}{2}\right\}
$$

$$
\delta(a)=\min _{\substack{n, m) \in M \\ m \neq 0}}\left|1+\frac{2 n \theta_{n}+\theta_{n}^{2}}{n^{2}-(a m)^{2}}\right| .
$$

Отметим, что $\delta(a) \neq 0$, поскольку $(n, m) \in M$. Из (17) следует, что найдется $a_{1} \in \mathbb{N}$ такое, что для всех натуральных $a \geqslant a_{1}$ при $m \neq 0$ имеет место оценка

$$
\left|\frac{2 n \theta_{n}+\theta_{n}^{2}}{n^{2}-(a m)^{2}}\right|<\frac{1}{2} \text {. }
$$

Поэтому $\delta(a)>1 / 2$ при $a \geqslant a_{1}$ и

$$
\begin{aligned}
I & \leqslant \sum_{n=1}^{\infty} n^{-2 \alpha}+2^{\alpha} \sum_{(n, m) \in M}\left(n^{2}-(a m)^{2}\right)^{-\alpha} \\
& \leqslant \sum_{n=1}^{\infty} n^{-2 \alpha}+2^{\alpha} \sum_{n \neq m}\left(n^{2}-m^{2}\right)^{-\alpha} \leqslant \sum_{n=1}^{\infty} n^{-2 \alpha}+2^{\alpha+1}\left(\sum_{n=1}^{\infty} n^{-\alpha}\right)^{2} .
\end{aligned}
$$

Отсюда и из (16) следует (15). Лемма доказана.

Из леммы 1 вытекает равномерная сходимость соответствующего ряда и то, что $A^{-1} v \in C(\Omega) \quad \forall v \in N_{3}$.

Лемма 2. Oператор $A^{-1}: N_{3} \rightarrow N_{3}$ вполне непрерывен.

ДокАЗАТЕльство. При доказательстве леммы 1 была установлена сходимость ряда

$$
I=\sum_{(n, m) \in M}\left|\lambda_{n}^{2}-(a m)^{2}\right|^{-\alpha}
$$

Положив $\alpha=2$, получим утверждение леммы. Лемма доказана.

Лемма 3. Собственные значения $\left.A\right|_{N_{3}}$ имеют конечную кратность.

ДокАЗАТЕЛЬСтво. Рассмотрим уравнение

$$
\left(n+\theta_{n}\right)^{2}-(a m)^{2}=\lambda .
$$

Оно равносильно равенству

$$
(n-a m)(n+a m)=\lambda-2 n \theta_{n}-\theta_{n}^{2} .
$$

Из (11) следует, что правая часть в этом равенстве ограничена и может принимать лишь конечное число целых значений. Теперь заметим, что при $k \in \mathbb{Z} \backslash\{0\}$ уравнение

$$
(a n-b m)(a n+b m)=k
$$

имеет не более конечного числа решений $(n, m) \in \mathbb{N} \times \mathbb{Z}_{+}$, которые получаются перебором всех цельх делителей $k$.

На подпространстве $N_{3}$ множитель $a n-b m \neq 0$. Поэтому при $k=0$ уравнение (18) решений не имеет. Лемма доказана. 
3. Доказательство теоремы 1. Рассмотрим конечномерное пространство $E_{n}=$ $N(A) \oplus N_{2 n} \oplus N_{3 n}$, где $N_{2 n}$ и $N_{3 n}$ есть линейные оболочки множеств

$$
\left\{\varphi_{k a}(x) \cos (k t), \varphi_{k a}(x) \sin (k t) \mid k \in \mathbb{N}, 1 \leqslant k \leqslant n\right\}
$$

и

$$
\left\{\varphi_{k}(x) \cos (\ell t), \varphi_{k}(x) \sin (\ell t) \mid(k, \ell) \in M, k, \ell \leqslant n\right\}
$$

соответственно.

Рассмотрим на $E_{n}$ функционал энергии

$$
F(v)=\frac{1}{2}(A v, v)+\frac{1}{p} \int_{\Omega}|v|^{p} d x d t-\int_{\Omega} f v d x d t, \quad v \in E_{n} .
$$

Легко видеть, что

$$
\lim _{\|v\| \rightarrow \infty} F(v)=+\infty
$$

Доказательство теоремы будет состоять из двух этапов:

I) доказательство существования критической точки $F$ на $E_{n}$;

II) предельный переход.

Этап I. Доказательство существования критической точки функционала $F$ на $E_{n}$ проведем, опираясь на следующую лемму из работы [10].

ЛЕмма 4. Пусть конечномерное пространство Е представлено в виде прямой суммы попарно ортогональных подпространств: $E=V_{1} \oplus V_{2} \oplus V_{3}$. Пусть $S$ есть сфера в $E$ с иентром в нуле. Пусть функиионал $F: E \rightarrow \mathbb{R}$ удовлетворяет следующим условиям:

1) $F \in C^{1}(E, \mathbb{R})$;

2) $\lim _{\|v\|_{E} \rightarrow \infty} F(v)=\infty$;

3) $F(v) \leqslant d \quad \forall v \in S \cap\left(V_{1} \oplus V_{2}\right)$;

4) $F(v)>d \quad \forall v \in V_{3}$;

5) $F(v)>c \quad \forall v \in V_{2} \oplus V_{3}$.

Следовательно, существует критическая точка $v_{0} \in E$ функиионала $F$ такая, что $F\left(v_{0}\right) \in[c, d]$.

Для любого $c \in \mathbb{R}$ обозначим $G_{c}$ и $L_{c}$ подпространства $E_{n}$, являющиеся линейной оболочкой тех собственных функций (12) оператора $A$, собственные значения которых не меньше и меньше соответственно числа $c$.

Для любого $c \in \mathbb{R}$ и любого $v \in G_{c}$

$$
F(v) \geqslant \frac{1}{2} c\|v\|^{2}+\frac{1}{\beta_{0} p}\|v\|_{p}^{p}-\frac{1}{\alpha_{0}}\|f\| \cdot\|v\| \geqslant C_{5} \cdot\|v\|^{p}-\frac{1}{2}|c| \cdot\|v\|^{2}-C_{5}^{\prime} d_{1}\|v\| .
$$

Здесь константы $C_{5}, C_{5}^{\prime}$ не зависят от $n, c$ и $a$. Обозначим

$$
m\left(c, d_{1}\right)=\min _{[0,+\infty)} h(\tau)-1,
$$

где

$$
h(\tau)=C_{5} \tau^{\rho}-\frac{1}{2}|c| \cdot \tau^{2}-C_{5}^{\prime} d_{1} \cdot \tau .
$$


Тогда

$$
F(v)>m\left(c, d_{1}\right) \quad \forall v \in G_{c} .
$$

Число $m\left(c, d_{1}\right)$ не зависит от $n$ и $a$.

Возьмем $v \in N_{3 n}$. Пусть $a_{k l}, b_{k l}, c_{k}$ есть коэффициенты $\Phi$ урье $v$ по системе (12):

$$
\begin{aligned}
a_{k l} & =\frac{1}{\sqrt{\pi}} \int_{\Omega} \rho(x) \varphi_{k}(x) \cos (\ell t) v(x, t) d x d t, \\
b_{k l} & =\frac{1}{\sqrt{\pi}} \int_{\Omega} \rho(x) \varphi_{k}(x) \sin (\ell t) v(x, t) d x d t, \\
c_{k} & =\frac{1}{\sqrt{2 \pi}} \int_{\Omega} \rho(x) \varphi_{k}(x) v(x, t) d x d t .
\end{aligned}
$$

Обозначим

$$
\|v\|_{s}=\left[\sum_{k=1}^{n} \lambda_{k}^{2 s} c_{k}^{2}+\sum_{k, l=1}^{n}\left|\lambda_{k}^{2}-(a \ell)^{2}\right|^{s}\left(a_{k l}^{2}+b_{k l}^{2}\right)\right]^{1 / 2} .
$$

Из оценки (15) и интерполяционного неравенства при $a \geqslant a_{1}$ следует оценка (см. [5])

$$
\|v\|_{p} \leqslant C_{6}\|v\|_{\beta} \quad \forall v \in N_{3 n}
$$

где $\beta=s(p-2) / p$. Если $s \in(1, p /(p-2))$, то

$$
\beta \in(0,1) .
$$

Константа $C_{6}$ не зависит от $n, v$ и $a$.

Лемма 5. Для любого $е \in \mathbb{R}$ существует $a_{0}=a_{0}\left(e, d_{1}\right) \in \mathbb{N}$ такое, ито если $a \geqslant a_{0}$ и $а \in \mathbb{N}$, то для любого $v \in\left\{u \in L_{0} \mid\|v\|_{\beta}=1\right\}$ выполняется неравенство

$$
F(v) \leqslant e .
$$

ДокАЗАТЕЛЬСтво. Пусть $v \in L_{0}$ такое, что $\|v\|_{\beta}=1$, и пусть $a_{k l}, b_{k l}, c_{k}$ есть коэффициенты Фурье функции $v$, вычисленные по формулам (21). Тогда

$$
\begin{aligned}
F(v) & \leqslant-\frac{1}{2} \sum_{k, l=1}^{n}\left|\lambda_{k}^{2}-(a \ell)^{2}\right| \cdot\left(a_{k l}^{2}+b_{k l}^{2}\right)+\frac{1}{\alpha_{0} p}\|v\|_{p}^{p}+C_{7} d_{1}\|v\| \\
& \leqslant-\frac{1}{2}|\lambda|^{1-\beta} \sum_{k, l=1}^{n}\left|\lambda_{k}^{2}-(a \ell)^{2}\right|^{\beta}\left(a_{k l}^{2}+b_{k l}^{2}\right)+\frac{C_{6}^{p}}{\alpha_{0} p}+C_{8} d_{1} \\
& =-\frac{1}{2}|\lambda|^{1-\beta}+\frac{C_{6}^{p}}{\alpha p}+C_{8} d_{1},
\end{aligned}
$$

где $\lambda$ есть наибольшее отрищательное собственное значение оператора $A$. Из (13) следует, что

$$
\lambda=(n-a m)(n+a m)+2 n \theta_{n}+\theta_{n}^{2} \leqslant-a+2 n \theta_{n}+\theta_{n}^{2} .
$$


Отсюда и из (11) выведем, что

$$
\lim _{a \rightarrow+\infty} \lambda=-\infty
$$

Из этого равенства и (25), учитывая (23), нетрудно получить утверждение леммы. Лемма доказана.

Проверим вьполнение условий леммы 4. Обозначим $E=E_{n}, V_{1}=L_{\lambda}, V_{3}=G_{0}$. Пусть $V_{2}$ есть линейная оболочка собственных векторов оператора $A$ с собственньм значением $\lambda$. Из леммы 3 следует, что $\operatorname{dim} V_{2}<\infty$. Обозначим $S=\left\{v \in V_{1}+V_{2} \mid\|v\| \|_{\beta}=1\right\}$.

Возьмем произвольное $c<m\left(0, d_{1}\right)$. Обозначим $\gamma\left(c, d_{1}\right)=\min \left(c, m\left(\lambda, d_{1}\right)\right)-1$. Из (20) следует, что

$$
F(v)>m\left(0, d_{1}\right)>c \quad \forall v \in V_{3} .
$$

Из леммы 5 следует, что существует $a_{0}=a_{0}\left(c, d_{1}\right)$ такое, что если $a \geqslant a_{0}$ и $a \in \mathbb{N}$, то

$$
F(v) \leqslant c \quad \forall v \in S .
$$

Из неравенства (20) выведем также, что

$$
F(v)>m\left(\lambda, d_{1}\right)>\gamma\left(c, d_{1}\right) \quad \forall v \in V_{2} \oplus V_{3}
$$

Условие 1) леммы 4 очевидно вьполнено. Условие 2) следует из (19). Таким образом, все условия леммы 4 вьполнены. Из нее следует, что существует критическая точка $v_{n} \in E_{n}$ функционала $F$ на $E_{n}$ такая, что $F\left(v_{n}\right) \in\left[\gamma\left(c, d_{1}\right), c\right]$. Следовательно,

$$
\begin{gathered}
\left(A v_{n}, \omega\right)+\int_{\Omega}\left|v_{n}\right|^{p-2} v_{n} \omega d x d t-\int_{\Omega} f \omega d x d t=0 \quad \forall \omega \in E_{n}, \\
\gamma\left(c, d_{1}\right) \leqslant \frac{1}{2}\left(A v_{n}, v_{n}\right)+\frac{1}{p} \int_{\Omega}\left|v_{n}\right|^{p} d x d t-\int_{\Omega} f v_{n} d x d t \leqslant c .
\end{gathered}
$$

Этап II. Если (26) умножить на $1 / 2$, положить в нем $\omega=v_{n}$ и полученное равенство вычесть из (27), то получим

$$
-\gamma\left(c, d_{1}\right) \geqslant\left(\frac{1}{2}-\frac{1}{p}\right) \int_{\Omega}\left|v_{n}\right|^{p} d x d t-\frac{1}{2} \int_{\Omega} f v_{n} d x d t \geqslant-c .
$$

Следовательно,

$$
\left\|v_{n}\right\|_{p} \leqslant C_{9}
$$

И

$$
\left\|v_{n}\right\|_{p}^{p}+C_{10}\left\|v_{n}\right\|_{p} \geqslant|c| \cdot C_{11}
$$

где положительные константы $C_{9}, C_{10}, C_{11}$ не зависят от $n$.

Отсюда вытекает, что найдется подпоследовательность, которую также обозначим $v_{n}$, такая, что $v_{n} \rightarrow v$ слабо в $L_{p}(\Omega)$ и $\left|v_{n}\right|^{p-2} v_{n} \rightarrow h$ слабо в $L_{q}(\Omega)$.

Докажем, что $v$ есть обобщенное решение задачи $\left(1^{\prime}\right)-\left(3^{\prime}\right)$. Для этого представим $v_{n}=v_{1 n}+v_{2 n}+v_{3 n}, v=v_{1}+v_{2}+v_{3}$, где $v_{1}, v_{1 n} \in N_{1}, v_{2}, v_{2 n} \in N_{2}$ и $v_{3}, v_{3 n} \in N_{3}$.

Легко видеть, что $v_{k n} \rightarrow v_{k}$ слабо в $L_{2}(\Omega)$. Не трудно показать, что $v_{2} \in D(A)$ и $A v_{2 n} \rightarrow A v_{2}$ слабо в $L_{2}(\Omega)$. 
Пусть $a_{k l}^{n}, b_{k l}^{n}, c_{k}^{n}$ и $a_{k l}^{0}, b_{k l}^{0}, c_{k}^{0}$ есть коэффициенты Фурье функций $v_{n}$ и $v$ соответственно. Подставим, как в [5], в равенство (26)

$$
\omega=\frac{1}{\sqrt{\pi}} \sum_{\left|\mu_{k m}\right| \geqslant R} \operatorname{sgn}\left(\mu_{k m}\right) \varphi_{k}(x)\left(a_{k l}^{n} \cos (l t)+b_{k l}^{n} \sin (l t)\right)+\frac{1}{\sqrt{2 \pi}} \sum_{k \geqslant \sqrt{R}} c_{k}^{n} \varphi_{k}(x)
$$

где $R>2 b_{1}+b_{1}^{2}$. Используя оценки $(22),(28)$ и неравенство Гёльдера, получим

$$
J_{R} \leqslant C_{12}\|\omega\|_{\beta} \leqslant \frac{C_{13}}{R^{(1-\beta) / 2}} \sqrt{J_{R}}
$$

где

$$
J_{R}=\sum_{\left|\mu_{k m}\right| \geqslant R}\left|\mu_{k m}\right|\left(\left(a_{k l}^{n}\right)^{2}+\left(b_{k l}^{n}\right)^{2}\right)+\sum_{k \geqslant \sqrt{R}} \lambda_{k}^{2}\left(c_{k}^{n}\right)^{2} .
$$

Таким образом,

$$
J_{R} \leqslant \frac{C_{13}^{2}}{R^{1-\beta}}
$$

где константа $C_{13}$ не зависит от $n$. Из последнего неравенства следует, что

$$
\begin{gathered}
\lim _{n \rightarrow \infty}\left(\sum_{\substack{a, k \neq b m \\
m \geqslant 1}} \mu_{k m}\left(\left(a_{k m}^{n}\right)^{2}+\left(b_{k m}^{n}\right)^{2}\right)+\sum_{k=1}^{\infty} \lambda_{k}^{2}\left(c_{k}^{n}\right)^{2}\right) \\
=\sum_{\substack{a k \neq b m \\
m \geqslant 1}} \mu_{k m}\left(\left(a_{k m}^{0}\right)^{2}+\left(b_{k m}^{0}\right)^{2}\right)+\sum_{k=1}^{\infty} \lambda_{k}^{2}\left(c_{k}^{0}\right)^{2} .
\end{gathered}
$$

Перейдем в равенстве (26) к пределу при $n \rightarrow \infty$ с фиксированным $\omega \in E_{n_{0}}$ :

$$
\left(A v_{2}, \omega\right)+\left(v_{3}, A \omega\right)+\int_{\Omega} h \omega d x d t-\int_{\Omega} f \omega=0
$$

Отсюда следует, что существует $A u_{3} \in L_{q}(\Omega)$. Докажем, что $h=|u|^{p-2} u$, воспользовавшись методом монотонности (см. [11]).

Для любого $u \in L_{p}(\Omega) \cap D(A)$ из (14) следует неравенство

$$
\left(A u_{2}-A v_{2 n}, u_{2}-v_{2 n}\right)+\int_{\Omega}\left(|u|^{p-2} u-\left|v_{n}\right|^{p-2} v_{n}\right)\left(u-v_{n}\right) d x d t \geqslant 0
$$

Положим в $(26) w=v_{n}$ и, воспользовавшись $(31)$, перейдем к пределу при $n \rightarrow \infty$ :

$$
\begin{aligned}
\lim _{n \rightarrow \infty}\left[\left(A v_{2 n}, v_{2 n}\right)+\int_{\Omega}\left|v_{n}\right|^{p} d x d t\right] \\
=\int_{\Omega} f v d x d t-\left(\sum_{\substack{a k \neq b m \\
m \geqslant 1}} \mu_{k m}\left(\left(a_{k m}^{0}\right)^{2}+\left(b_{k m}^{0}\right)^{2}\right)+\sum_{k=1}^{\infty} \lambda_{k}^{2}\left(c_{k}^{0}\right)^{2}\right) .
\end{aligned}
$$


Подставим в $(32) \omega=v_{n}$ и устремим $n \rightarrow \infty$ :

$$
\begin{aligned}
& \left(A v_{2}, v_{2}\right)+\int_{\Omega} h v d x d t \\
& \quad=\int_{\Omega} f v d x d t-\left(\sum_{\substack{a k \neq b m \\
m \geqslant 1}} \mu_{k m}\left(\left(a_{k m}^{0}\right)^{2}+\left(b_{k m}^{0}\right)^{2}\right)+\sum_{k=1}^{\infty} \lambda_{k}^{2}\left(c_{k}^{0}\right)^{2}\right) .
\end{aligned}
$$

Из последних двух равенств следует

$$
\lim _{n \rightarrow \infty}\left[\left(A v_{2 n}, v_{2 n}\right)+\int_{\Omega}\left|v_{n}\right|^{p} d x d t\right]=\left(A v_{2}, v_{2}\right)+\int_{\Omega} h v d x d t
$$

Теперь в неравенстве (33) можно перейти к пределу при $n \rightarrow \infty$ :

$$
\left(A\left(u_{2}-v_{2}\right), u_{2}-v_{2}\right)+\int_{\Omega}\left(|u|^{p-2} u-h\right)(u-v) d x d t \geqslant 0 .
$$

Взяв $u=v+\lambda \phi$ и устремив $\lambda$ к нулю, после стандартных рассуждений получим $h=|v|^{p-2} v$. Из (32) следует, что $v$ есть обобщенное решение задачи $\left(1^{\prime}\right)-\left(3^{\prime}\right)$. Неравенство $\|v\|_{p} \geqslant d_{2}$ вытекает из (29) и (35) при достаточно большом $|c|$. Теорема доказана.

ЗАмЕчАнИЕ. При доказательстве теоремы мы не использовали однородность нелинейного слагаемого. Рассмотрим уравнение

$$
\rho(x) u_{t t}-\left(\rho(x) u_{x}\right)_{x}+g(x, t, u)=0
$$

Пусть вьполнены следующие условия (см. [5]):

$$
\begin{aligned}
& g(x, t, u) \text { не убывает по } u \text { и } T \text {-периодична по } t, \text { где } T=2 \pi / a, a \in \mathbb{N} ; \\
& g(x, t, u) \text { непрерьвна при }(x, t, u) \in[0, \pi] \times \mathbb{R} \times \mathbb{R} .
\end{aligned}
$$

Существуют положительные константы $A_{1}, A_{2}, A_{3}, A_{4}, \delta$ такие, что при всех $(x, t, u)$ $\in[0, \pi] \times \mathbb{R} \times \mathbb{R}$ вьполнено неравенство

$$
A_{1}|u|^{p-1}+A_{2} \geqslant|g(x, t, u)| \geqslant A_{3}|u|^{p-1}-A_{4} \quad \text { и } \quad \frac{A_{3}}{2} \geqslant \frac{A_{1}}{p}+\delta .
$$

ТЕОрема 2. Предположим выполнены условия (36)-(38), функиия $\rho(x)$ удовлетворяет условиям (4), (5). Тогда для любого $d>0$ существует $a_{0}=a_{0}\left(d, A_{1}, A_{2}\right.$, $\left.A_{3}, A_{4}, \delta\right) \in \mathbb{N}$ такое, что при любом $a \geqslant a_{0}$ и $a \in \mathbb{N}$ задача $\left(1^{\prime \prime}\right),(2)$, (3) имеет обобщенное решение $u \in L_{p}(\Omega)$ такое, что $\|u\|_{p} \geqslant d$.

Нетрудно проверить, что доказательство теоремы 2 полностью повторяет доказательство теоремы 1 с небольшими видоизменениями. 


\section{4. Волновое уравнение с постоянными коэффициентами и с однородными} граничными условиями. Рассмотрим задачу

$$
\begin{gathered}
u_{t t}-u_{x x}+g(x, t, u)=0, \quad 0<x<\pi, \quad t \in \mathbb{R}, \\
u(0, t)-h_{1} u_{x}^{\prime}(0, t)=0, \quad u(\pi, t)+h_{2} u_{x}^{\prime}(\pi, t)=0, \quad t \in \mathbb{R}, \\
u(x, t+T)=u(x, t), \quad 0<x<\pi, \quad t \in \mathbb{R} .
\end{gathered}
$$

Здесь $h_{1}>0, h_{2}>0$ и $1 /\left(h_{1} \cdot h_{2}\right)+1 / 2 \notin \mathbb{N}, T=2 \pi / a$, где $a \in \mathbb{N}$.

Результаты, полученные для задачи $\left(1^{\prime \prime}\right),(2),(3)$, будут справедливы и для задачи (39)-(41). Это связано с тем, что линейные части этих задач имеют похожий спектр. Обозначим через $A$ замыкание по графику в $L_{2}(\Omega)$ оператора Даламбера $\partial_{t t}-\partial_{x x}$, определенного на функциях из $C^{\infty}(\Omega)$, удовлетворяющих условиям $(40),(41)$.

В этом пункте мы будем иметь дело с обьчньп пространством $L_{p}(\Omega)($ вес $\rho(x) \equiv 1)$.

Решениями соответствующей задачи Штурма-Лиувилля

$$
\begin{gathered}
\varphi_{x x}+\lambda^{2} \varphi=0, \\
\varphi(0)-h_{1} \varphi^{\prime}(0)=0, \quad \varphi(\pi)+h_{2} \varphi^{\prime}(\pi)=0,
\end{gathered}
$$

является полная ортонормированная в $L_{2}([0, \pi])$ система функций

$$
\varphi_{n}(x)=C_{n}\left(\sin \lambda_{n} x+\lambda_{n} h_{1} \cos \lambda_{n} x\right),
$$

где $\lambda_{n}=(n-1)+\theta_{n}, C_{n}=C / \lambda_{n}+O(1), C>0$. Таким образом, $\varphi_{n}(x)$ равномерно ограничены в $L_{\infty}(\Omega)$. В [12] доказано, что существуют положительные константы $b_{0}$ и $b_{1}$ такие, что вьполнено условие (11).

Собственные функции оператора $A$ составляют следующую полную ортонормированную в $L_{2}(\Omega)$ систему функций:

$$
\left\{\frac{1}{\sqrt{T}} \varphi_{n}(x), \sqrt{\frac{2}{T}} \varphi_{n}(x) \cos (a m t), \sqrt{\frac{2}{T}} \varphi_{n}(x) \sin (a m t)\right\}_{m, n \in \mathbb{N}} .
$$

Собственные значения $A$ имеют вид

$$
\mu_{n m}=\lambda_{n}^{2}-(a m)^{2}, \quad n \in \mathbb{N}, \quad m \in \mathbb{Z}_{+} .
$$

Обозначим

$$
\begin{aligned}
& M=\left\{(n, m) \mid n \in \mathbb{N}, m \in \mathbb{Z}_{+}, \lambda_{n} \neq a m,(n-1) \neq a m\right\}, \\
& \Lambda_{1}=\left\{\varphi_{n}(x) \cos ((n-1) t), \varphi_{n}(x) \sin ((n-1) t) \mid \frac{n-1}{a} \in \mathbb{Z}_{+}\right\}, \\
& \Lambda_{2}=\left\{\varphi_{n}(x) \cos (a m t), \varphi_{n}(x) \sin (a m t) \mid(n, m) \in M\right\} .
\end{aligned}
$$

Пусть $N_{1}=N(A)$ (ядро $\left.A\right), N_{2}=\overline{L\left(\Lambda_{1}\right)}, N_{3}=\overline{L\left(\Lambda_{2}\right)}$. Справедливы следуюшие свойства $A$ (см. [12]):

a) $A$ самосопряжен в $L_{2}(\Omega), R(A)$ замкнут в $L_{2}(\Omega)$;

б) $R(A)=N(A)^{\perp}, L_{2}(\Omega)=N(A) \oplus R(A)$;

в) на $N_{2}$ собственные значения $A$ равны $2(n-1) \theta_{n}+\theta_{n}^{2}>c_{0}>0$, где $c_{0}=\min \left(b_{0}, \theta_{1}^{2}\right)$;

г) $\operatorname{dim} N(A)<\infty$;

д) собственные значения $A$ на $N_{3}$ имеют конечную кратность.

Обозначим $D_{0}=\left\{u(x, t) \in C^{\infty}(\Omega) \mid u(x, t)\right.$ удовлетворяет $(40),(41)$ и $u_{t}(x, 0)=$ $\left.u_{t}(x, T)\right\}$. 
ОПРЕДЕЛЕНИЕ. Функция $u \in L_{p}$ называется обобщенным решением задачи (39)(41), если

$$
\int_{\Omega} u\left(\varphi_{t t}-\varphi_{x x}\right) d x d t+\int_{\Omega} g(x, t, u) \varphi d x d t=0 \quad \forall \varphi \in D_{0}
$$

ТЕОремА 3. Предположим выполнены условия (36)-(38). Тогда для любого $d>0$ существует $a_{0}=a_{0}(d) \in \mathbb{N}$ такое, ито при любом $a \geqslant a_{0}$ и $а \in \mathbb{N}$ задача (39)-(41) имеет обобщенное решение $u \in L_{p}(\Omega)$ такое, что $\|u\|_{p} \geqslant d$.

Нетрудно убедиться, что благодаря перечисленньм вьше свойствам $A$, доказательство теоремы 3 полностью повторяет доказательство теоремы 1.

\section{СПИСОК ЦИТИРОВАННОЙ ЛИТЕРАТУРЫ}

[1] Barbu V., Pavel N.H. Periodic solutions to nonlinear one-dimensional wave equation with $x$-dependent coefficients // Trans. Amer. Math. Soc. 1997. V. 349. № 5. P. 2035-2048.

[2] Rabinowitz P. Free vibration for a semilinear wave equation // Comm. Pure Appl. Math. 1980. V. 33. № 3. P. 667-689.

[3] Bahri A., Brézis H. Periodic solution of a nonlinear wave equation // Proc. Roy. Soc. Edinburgh Sect. A. 1980. V. 85. №3-4. P. 313-320.

[4] Плотников П.И. Существование счетного множества периодических решений задачи о вынужденных колебаниях для слабо нелинейного волнового уравнения // Матем. сб. 1988. T. 136 (178). № 4 (8). C. 546-560.

[5] Feireisl E. On the existence of periodic solutions of a semilinear wave equation with a superlinear forcing term // Czechoslovak Math. J. 1988. V. 38 (113). №1. P. 78-87.

[6] Brézis H., Nirenberg L. Forced vibrations for a nonlinear wave equation // Comm. Pure Appl. Math. 1978. V. 31. № 1. P. 1-30.

[7] Рудаков И. А. Нелинейные колебания струны // Вестн. МГУ. Сер. 1. Матем., мех. 1984. № 2. С. $9-13$.

[8] Похожаев С. И. О методе расслоения решения нелинейных краевых задач // Тр. МИАН. 1990. T. 192. C. $146-163$.

[9] Рудаков И. А.Периодическое по времени решение нелинейного волнового уравнения с непостоянными коэффициентами // Фундамент. и прикладная матем. 2002. № 3. С. 42-49.

[10] Feireisl E. Time periodic solutions to a semilinear wave equation // Nonlinear Anal. 1988. V. 12. P. 279-290.

[11] Лионс ЖК.-Л. Некоторые методы решения нелинейных краевых задач. М.: Едиториал УРСС, 2002.

[12] Рудаков И.А. Периодическое по времени решение уравнения вынужденных колебаний струны с однородными граничными условиями // Дифференц. уравнения. 2003. Т. 39. № 12 . C. $1-6$. 